\title{
EFEITO DA NATUREZA DO ELETRÓLITO E DA FORÇA IÔNICA NA ENERGIA LIVRE DA REAÇÃO DE ADSORÇÃO DE NÍQUEL EM SOLOS ${ }^{(1)}$
}

\author{
Cindy Silva Moreira Iglesias ${ }^{(2)}$, José Carlos Casagrande ${ }^{(3)} \&$ Luis \\ Reynaldo Ferracciú Alleoni ${ }^{(4)}$
}

\begin{abstract}
RESUMO
A adsorção é o principal processo responsável pelo acúmulo de metais pesados na superfície dos colóides do solo. O conhecimento detalhado desse fenômeno pode fornecer subsídios para o aprimoramento das práticas de remediação de solos contaminados. Avaliou-se a energia livre $\left(\Delta G_{0}\right)$ de adsorção de $\mathrm{Ni}$ em amostras superficiais $(0,0-0,2 \mathrm{~m})$ e subsuperficiais (na maior expressão do horizonte $B$ ) de um Latossolo Vermelho acriférrico típico textura argilosa (LVwf) e de um Nitossolo Vermelho eutroférrico textura muito argilosa (NVef), utilizando-se soluções de $\mathrm{NaCl}$ e $\mathrm{CaCl}_{2}$ em três forças iônicas $\left(1,0,0,1 \mathrm{e} 0,01 \mathrm{~mol} \mathrm{~L}^{-1}\right)$. As amostras de solo receberam $2,5,10,20,30,40,50 \mathrm{e} 70 \mathrm{mg} \mathrm{dm}^{-3} \mathrm{de} \mathrm{Ni}$, na proporção solo:solução de 1:10. A adsorção de Ni pelos solos foi espontânea, visto que $a \Delta G_{0}$ apresentou valores negativos em todas as concentrações estudadas. Os valores de $\Delta \mathrm{G}_{0}$ diminuíram com o aumento da dose de Ni adicionada. O NVef apresentou maior $\Delta \mathrm{G}_{0}$ que o LVwf devido, principalmente, às suas características químicas e mineralógicas. Os horizontes superficiais apresentaram, em geral, maior $\Delta G_{0}$ em relação aos subsuperficiais, em razão do elevado teor de matéria orgânica encontrado em superfície. A $\Delta \mathbf{G}_{0}$ foi maior para as menores forças iônicas do meio, tanto para $\mathrm{CaCl}_{2}$ quanto para $\mathrm{NaCl}$.
\end{abstract}

Termos de indexação: pH, metal pesado, termodinâmica.

\footnotetext{
(1) Trabalho extraído do Projeto de Iniciação Científica do primeiro autor, financiado pela FAPESP. Recebido em março de 2006 e aprovado em maio de 2007.

(2) Doutoranda em Ciências, Universidade de São Paulo CENA/USP. E-mail: ciglesias@cena.usp.br

(3) Professor Adjunto do Departamento de Recursos Naturais e Proteção Ambiental Centro de Ciências Agrárias, Universidade Federal de São Carlos - UFSCar. Caixa Postal 153, CEP 13600-970 Araras (SP). E-mail: bighouse@power.ufscar.br

(4) Professor Associado do Departamento de Solos e Nutrição de Plantas, Escola Superior de Agricultura Luiz de Queiroz USP/ESALQ. Caixa Postal 9, CEP 13418-900 Piracicaba (SP). E-mail: lrfalleo@esalq.usp.br
} 


\title{
SUMMARY: EFFECT OF ELECTROLYTE NATURE AND IONIC STRENGTH IN THE FREE ENERGY OF NICKEL ADSORPTION REACTION INSOILS
}

\begin{abstract}
The adsorption is the main process responsible for the accumulation of heavy metals in the surface of soil colloids. Detailed knowledge of this phenomenon can contribute to improve the remediation practices for contaminated soil. The free energy $\left(\Delta G_{0}\right)$ of $\mathrm{Ni}$ adsorption was evaluated in surface $(0.0-0.2 \mathrm{~m})$ and subsurface (in the maximum expression of $B$ horizon) samples of a clayey Rhodic Acrudox (RA) and a very clayey Eutric Kandiudalf (EK). Ni was added $\left(2,5,10,20,30,40,50\right.$, and $\left.70 \mathrm{mg} \mathrm{dm}^{-3}\right)$, in a 1:10 soil:solution ratio. Two background electrolytes ( $\mathrm{NaCl}$ and $\left.\mathrm{CaCl}_{2}\right)$ and three ionic strengths - IS (1.0; 0.1 and $\left.0.01 \mathrm{~mol} \mathrm{~L}^{-1}\right)$ were tested. The $\mathrm{Ni}$ adsorption reaction was spontaneous, since the $\Delta G_{0}$ values were negative in all concentrations. Values of $\Delta G_{0}$ decreased with the increasing $\mathrm{Ni}$ doses. $\Delta G_{0}$ was higher in the EK presented than in $R A$, mainly due to its chemical and mineralogical characteristics. In surface samples, $\Delta G_{0}$ was generally higher than in subsurface samples due to their higher organic matter contents. The $\Delta G_{0}$ was higher for solutions with lower IS, regardless of the electrolyte type.
\end{abstract}

Index terms: $p H$, heavy metal, thermodynamics.

\section{INTRODUÇÃO}

A adsorção de metais pesados em solos altamente intemperizados dos trópicos úmidos e a identificação dos fatores que mais influenciam sua mobilidade e biodisponibilidade podem oferecer subsídios para previsão de fitotoxidez e da possível contaminação do lençol freático por esses elementos.

O Ni está presente no solo a partir de rochas ígneas ultrabásicas (Uren, 1992). Os teores encontrados nos solos variam de acordo com o material de origem, que reflete as variações das zonas ecológicas (Rovers et al., 1983). No estado puro, dada sua grande resistência à corrosão, o Ni é empregado em indústrias de galvanoplastia como revestimento eletrolítico, servindo para proteger ligas de $\mathrm{Fe}$ e $\mathrm{Cu}$, a fim de melhorar as propriedades térmicas e mecânicas de numerosas ligas. Resíduos destas e de outras indústrias podem conter elevado conteúdo de Ni (Pombo et al., 1989), principalmente na camada superficial, de 0 a $20 \mathrm{~cm}$ (Oliveira et al., 2005).

O Ni é tóxico para diversas plantas, mas sua toxicidade pode ser atenuada na presença de $\mathrm{Ca}^{2+}$ ou até agravada pela presença de $\mathrm{Mg}^{2+}$ (Proctor \& Baker, 1994). Plantas cultivadas em solos contaminados por resíduos industriais que contêm $\mathrm{Ni}$ ou em solos que receberam resíduos orgânicos como lodo de esgoto (não tratado) são comuns e agravam ainda mais o problema.

Há alguns anos, o Ni passou a ser considerado elemento essencial para as plantas, embora seja requerido em quantidades muito pequenas (Camargo et al., 1989; Uren, 1992; Proctor \& Baker, 1994). A disponibilidade de Ni para as plantas é regulada, em grande parte, pelas reações de adsorção que ocorrem entre o elemento e as superfícies sólidas do solo. Essas reações são influenciadas pela capacidade de troca de cátions, pH, teor de argila e matéria orgânica (MO) no perfil, superfície específica, teor de óxidos, eletrólitosuporte, entre outras (Harter, 1983; Camargo et al., 1989; Pombo et al., 1989; Scheidegger et al., 1996; Teixeira et al., 2005).

Com base nos resultados de adsorção de Ni, podemse fazer algumas inferências, como o cálculo termodinâmico da energia livre das reações envolvidas $\left(\Delta \mathrm{G}_{0}\right)$. As mudanças na energia livre que ocorrem quando um elemento é adsorvido podem servir como medida da extensão ou da força que guia determinada reação (Singh, 1971). Seu sinal indica se a reação é espontânea ou não, em um sistema fechado, à temperatura e pressão constantes. Assim, valores negativos de energia livre significam que a reação é espontânea e exotérmica. O contrário pode ser concluído caso valores positivos de $\Delta \mathrm{G}_{0}$ sejam obtidos. Quanto maior a magnitude do valor de energia livre, maior a força de reação de determinado soluto pela superfície adsorvente (Alleoni \& Camargo, 1998).

Alguns pesquisadores estudaram a energia livre da reação de adsorção de $\mathrm{Cd}$ (Dias et al., 2003), $\mathrm{Cu}$ (Silveira et al., 1999) e B (Alleoni \& Camargo, 1998) em solos. No entanto, pesquisas relacionadas à $\Delta \mathrm{G}_{0}$ são escassas, sobretudo em se tratando de solos ácricos, que representam o extremo na escala de intemperização (Alleoni \& Camargo, 1994). Esses solos caracterizam-se pelo intenso processo de dessilicatização, resultando no acúmulo de óxidos de Fe (hematita e goethita) e Al (gibbsita), além da caulinita. O caráter ácrico aparece quando o solo apresenta CTC efetiva menor que $15 \mathrm{mmol}_{\mathrm{c}} \mathrm{kg}^{-1}$ de argila e $\mathrm{pH}\left(\mathrm{KCl} 1 \mathrm{~mol} \mathrm{~L}^{-1}\right)$ maior ou igual a 5,0, ou $\Delta \mathrm{pH}$ positivo ou nulo (Embrapa, 2000). Além disso, os solos são muito heterogêneos na sua constituição e seus atributos influenciam os fenômenos de maneira muito complexa (Alleoni, 2000). 
O objetivo deste trabalho foi estudar a energia livre da reação de adsorção de Ni como variável da concentração do metal, $\mathrm{pH}$, tipo de eletrólito-suporte e força iônica, em dois solos do Estado de São Paulo.

\section{MATERIAL E MÉTODOS}

Foram coletadas amostras de solo nas camadas de 0,0-0,2 e 1,0-1,4 m de um Latossolo Vermelho acriférrico típico (LVwf) textura argilosa e nas camadas de $0-0,2 \mathrm{~m}$ e $0,7-0,9 \mathrm{~m}$ de um Nitossolo Vermelho eutroférrico (NVef) textura muito argilosa, no município de Ribeirão Preto-SP $\left(21^{\circ} 17^{\prime} \mathrm{S}\right.$ e $47^{\circ}$ $81^{\prime}$ W), usado como referência, por apresentar menor grau de intemperização. Para as análises químicas, físicas e mineralógicas utilizou-se o método descrito por Camargo et al. (1986). O carbono orgânico foi determinado por oxidação da MO do solo com solução de dicromato de potássio em presença de ácido e titulação do excesso de dicromato com sulfato ferroso amoniacal; o $\mathrm{pH}$ foi medido em $\mathrm{H}_{2} \mathrm{O}$ e em $\mathrm{KCl}$ $1 \mathrm{~mol} \mathrm{~L}^{-1}$ na relação solo-solução 1:2,5; o ponto de efeito salino nulo (PESN) foi obtido pela titulação das amostras com $\mathrm{H}^{+}$e $\mathrm{OH}^{-}$em três concentrações de $\mathrm{KCl}$ (0,1; 0,01; e 0,001 $\mathrm{mol} \mathrm{L}^{-1}$ ) (Alleoni \& Camargo, 1994). O Fe livre foi determinado pelo método do bicarbonatoditionito-citrato, e o Fe e $\mathrm{Al}$ mal cristalizados, em ácido oxálico e seu sal de amônio. Os teores de óxidos $\left(\mathrm{SiO}_{2}\right.$, $\mathrm{Fe}_{2} \mathrm{O}_{3}$ e $\mathrm{Al}_{2} \mathrm{O}_{3}$ ) foram obtidos após ataque com ácido sulfúrico. $\mathrm{O} \Delta \mathrm{pH}$ foi calculado pela fórmula: $\Delta \mathrm{pH}=$ $\mathrm{pH} \mathrm{KCl}-\mathrm{pH} \mathrm{H}_{2} \mathrm{O}$.

A análise granulométrica foi feita pelo método da pipeta, e a determinação da superfície específica (SE), pelo método de Cihacek \& Bremmer (1979), com base na técnica de retenção de éter monoetílico do etilenoglicol (EMEG), proposto por Heilman et al. (1965). Nas análises mineralógicas qualitativas e quantitativas foram utilizadas amostras de argila previamente tratadas para eliminação da MO e dos óxidos de Fe. Com o auxílio da difração de raios $\mathrm{X}$, fez-se a determinação qualitativa nas amostras saturadas com $\mathrm{Mg}^{2+}$, glicoladas, e nas saturadas com $\mathrm{K}^{+}$submetidas às temperaturas de 25,350 e $550^{\circ} \mathrm{C}$. Os teores de caulinita e gibbsita foram obtidos por meio de análise térmica diferencial na fração argila desferrificada e isenta de MO.

Foram obtidas curvas de adsorção para cada amostra de solo após adição de 2, 5, 10, 20, 30, 40, 50 e $70 \mathrm{mg} \mathrm{dm}^{-3} \mathrm{de} \mathrm{Ni}$, em duplicata. Soluções de $\mathrm{NaCl}$ e $\mathrm{CaCl}_{2}$ foram utilizadas como eletrólito-suporte, nas concentrações de 1,0; 0,1; e $0,01 \mathrm{~mol} \mathrm{~L}^{-1}$. Amostras equivalentes a 2,0 $\mathrm{g}$ de terra fina seca ao ar foram agitadas por $24 \mathrm{~h}$ em tubos de polietileno, com $20 \mathrm{~mL}$ de solução de $\mathrm{NaCl}$ ou $\mathrm{CaCl}_{2}$ nas três concentrações, contendo as diferentes concentrações de Ni. O Ni na solução de equilíbrio foi determinado por espectrofotometria de absorção atômica, enquanto o teor de $\mathrm{Ni}$ adsorvido foi calculado subtraindo-se o $\mathrm{Ni}$ em equilíbrio na solução do Ni adicionado.

Com base nos resultados de adsorção versus $\mathrm{Ni}$ em equilíbrio e nos envelopes de adsorção de $\mathrm{Ni}$ (adsorção versus $\mathrm{pH}$ ), utilizando $\mathrm{NaCl}$ e $\mathrm{CaCl}_{2}$ como eletrólitos-suporte, nas três forças iônicas $(0,01 ; 0,1$; e 1,0 $\mathrm{mol} \mathrm{L}^{-1}$ ), foram calculados os valores de $\Delta \mathrm{G}_{0}$, de acordo com a seguinte equação termodinâmica: $\Delta \mathrm{G}_{0}=\mathrm{RT}$ (ln Ni equilíbrio - ln Ni adicionado), em que $\Delta \mathrm{G}_{0}=$ energia livre $\left(\mathrm{J} \mathrm{mol}^{-1}\right) ; \mathrm{R}=$ constante universal dos gases $\left(8,3 \mathrm{~J} \mathrm{~mol}^{-1} \mathrm{~K}^{-1}\right) ; \mathrm{e} \mathrm{T}=298^{\circ} \mathrm{K}$.

\section{RESULTADOS E DISCUSSÃO}

O LVwf apresentou baixa fertilidade, em conseqüência do avançado estadio de intemperismo do sistema. Na amostra coletada em superfície, a carga foi negativa devido ao efeito da MO (Quadro 1). Já em profundidade, em que a participação da MO foi menor, a carga líquida foi positiva, e o PESN foi maior que o $\mathrm{pH}$ dos solos. Esses resultados corroboram os valores obtidos nesse solo por Silveira et al. (1999), Dias et al. (2003) e Casagrande et al. (2004), em estudos envolvendo a adsorção de metais pesados. Diferentemente do Latossolo, o NVef apresentou elevada fertilidade, com predomínio de carga negativa nas duas profundidades estudadas, com destaque para a camada superficial, em razão do conteúdo mais elevado de MO. Como conseqüência, o NVef apresentou valores mais baixos de PESN e elevada SE, em ambas as profundidades.

O índice Ki, indicativo do grau de intemperização de solos, foi maior que 0,75 em todas as amostras, permitindo classificá-los como cauliníticos, de acordo com os critérios estabelecidos por Resende \& Santana (1988). No entanto, o NVef apresentou valores mais elevados de $\mathrm{Ki}(1,89$ e 1,74 em superfície e profundidade, respectivamente), o que denota menor estado de intemperização.

O LVwf apresentou concentrações mais elevadas de Fe total $\left(\mathrm{Fe}_{\mathrm{as}}\right)$, Fe livre $\left(\mathrm{Fe}_{\mathrm{d}}\right)$ e $\mathrm{Al}$ total $\left(\mathrm{Al}_{\mathrm{as}}\right)$, se comparado ao NVef. Apesar disso, não houve variação da percentagem de óxido de $\mathrm{Fe}$ na forma livre em relação ao conteúdo total deste óxido nas duas profundidades estudadas. Para o NVef, essa percentagem foi maior em profundidade do que em superfície (55 e $54 \%$, respectivamente). Em relação à percentagem de $\mathrm{Fe}$ na forma mal cristalizada ou amorfa, o NVef teve maior participação em relação ao conteúdo total de óxido de Fe (4 e 5 \%, em superfície e profundidade, respectivamente). Os óxidos de Fe e de $\mathrm{Al}$ exercem marcante influência nos atributos eletroquímicos de solos altamente intemperizados dos trópicos (Uren, 1992; Alleoni \& Camargo, 1995).

O PESN das camadas superficiais dos solos variou de 3,4 a 3,6, sendo menores que os valores de $\mathrm{pH}$ em água. Esses resultados estão de acordo com referências 
bibliográficas relacionadas a solos altamente intemperizados (Alleoni \& Camargo, 1994; Casagrande et al., 2002, 2004). Para as amostras subsuperficiais do LVwf, os valores de PESN foram mais elevados em razão, provavelmente, da pequena participação da MO e do predomínio de componentes com carga variável, como minerais oxídicos e caulinita (Naidu et al., 1994), cujos valores de PESN são mais elevados (em torno de 8,0 e 4,7, respectivamente), o que contribui para aumentar o PESN do solo (Miranda-Trevino \& Coles, 2003). Para o NVef não houve variação no PESN em ambas as profundidades, sendo baixo em ambas as situações. Raij \& Peech (1972) relataram que a presença de MO, sílica e minerais com carga permanente (minerais silicatados do tipo 2:1) diminui o PESN dos solos. Os diferentes valores desse atributo para as amostras de solos podem ajudar a explicar o efeito da força iônica na adsorção de Ni.
Os resultados de energia livre foram negativos para todas as amostras (Figuras 1 e 2), o que demonstra que a quantidade de Ni em equilíbrio na solução foi sempre inferior ao valor de Ni adicionado. Assim, a reação de adsorção de $\mathrm{Ni}$ foi termodinamicamente exotérmica e espontânea. Esses resultados estão de acordo com os obtidos para $\mathrm{Cu}$ em solos tropicais (Silveira et al., 1999) e Cd para as mesmas condições (Percival et al., 1999; Dias et al., 2003). Em estudos de $\Delta \mathrm{G}_{0}$ da reação de adsorção de $\mathrm{B}$ para diversos solos ácricos, Alleoni \& Camargo (1998) também observaram resultados semelhantes, apesar de o elemento não se tratar de um cátion. Os valores de $\Delta \mathrm{G}_{0}$, em módulo, foram sempre decrescentes com o aumento da concentração do Ni em solução (Figuras 1 e 2); nas doses menores de Ni, a amplitude de variação nos valores de $\Delta \mathrm{G}_{0}$ foi maior do que nas doses mais elevadas do metal.

Quadro 1. Atributos químicos, físicos e mineralógicos dos solos estudados

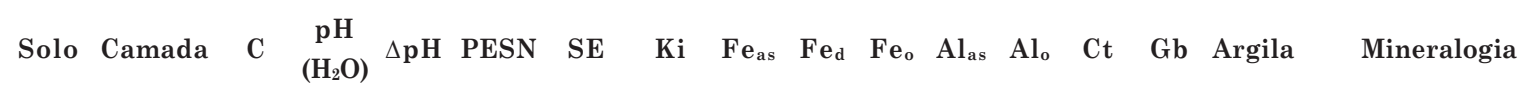

\begin{tabular}{|c|c|c|c|c|c|c|c|c|c|c|c|c|c|c|c|c|}
\hline \multirow[b]{2}{*}{ NVef } & \multirow{2}{*}{$\begin{array}{c}\mathrm{m} \\
0,0-0,2\end{array}$} & \multirow{2}{*}{$\begin{array}{l}\mathrm{g} \mathrm{kg}^{-1} \\
26\end{array}$} & \multirow[b]{2}{*}{5,7} & \multicolumn{4}{|c|}{$\mathrm{m}^{2} \mathrm{~g}^{-1}$} & \multicolumn{8}{|c|}{$\mathrm{g} \mathrm{kg}^{-1}$} & \multirow[b]{2}{*}{$\mathrm{Ct}>\mathrm{Gb}>\mathrm{VHE}$} \\
\hline & & & & $-0,9$ & 3,7 & 134 & 1,89 & 270 & 150 & 12 & 170 & 8 & 300 & 120 & 820 & \\
\hline \multirow{3}{*}{ LVwf } & $0,7-0,9$ & 7 & 6 & $-0,7$ & 3,6 & 151 & 1,74 & 280 & 150 & 14 & 200 & 7 & 570 & 100 & 700 & $\mathrm{Gt} \geq \mathrm{Hm}>\mathrm{Mh}>\mathrm{Mgn}$ \\
\hline & $0,0-0,2$ & 20 & 6,4 & $-0,9$ & 3,6 & 65 & 0,93 & 320 & 160 & 13 & 200 & 10 & 270 & 460 & 600 & $\mathrm{~Gb}>\mathrm{Ct}>\mathrm{VHE}$ \\
\hline & $1,0-1,4$ & 5 & 5,3 & 0,3 & 5,7 & 76 & 0,87 & 340 & 170 & 9 & 200 & 7 & 410 & 410 & 640 & $\mathrm{Hm}>\mathrm{Gt}>\mathrm{Mg}$ \\
\hline
\end{tabular}

NVef: Nitossolo Vermelho eutroférrico; LVwf: Latossolo Vermelho acriférrico típico; PESN: ponto de efeito salino nulo; SE: superfície específica; $\mathrm{Fe}_{\mathrm{as}}$ e $\mathrm{Al}_{\mathrm{as}}$ : óxidos de ferro e alumínio obtidos pelo ataque sulfúrico; $\mathrm{Fe}_{\mathrm{d}}$ e $\mathrm{Al}_{\mathrm{d}}$ : óxidos de ferro e alumínio "livres"; $\mathrm{Fe}_{\mathrm{o}}$ e $\mathrm{Al}_{\mathrm{o}}$ : óxidos de ferro e alumínio mal cristalizados; VHE: vermiculita com hidroxila entre camadas cloritizadas; Gt: goetita; Hm: hematita; Mh: maghemita; Mgn: magnetita; Ct: caulinita; Gb: gibbsita.

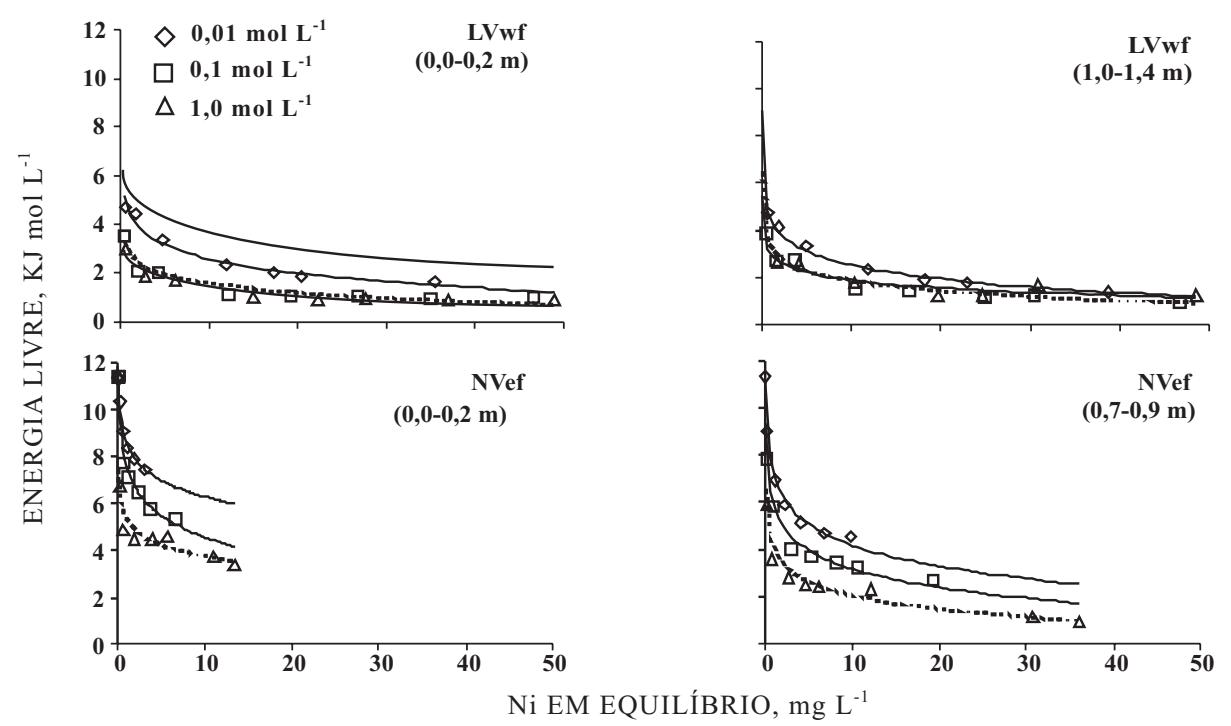

Figura 1. Energia livre da reação de adsorção de Ni para Latossolo Vermelho acriférrico (LVwf) e Nitossolo Vermelho eutroférrico (NVef), coletados em superfície e profundidade, utilizando $\mathrm{NaCl}$ nas forças iônicas 0,$01 ; 0,1 ; \mathrm{e} 1,0 \mathrm{~mol} \mathrm{~L}^{-1}$. 


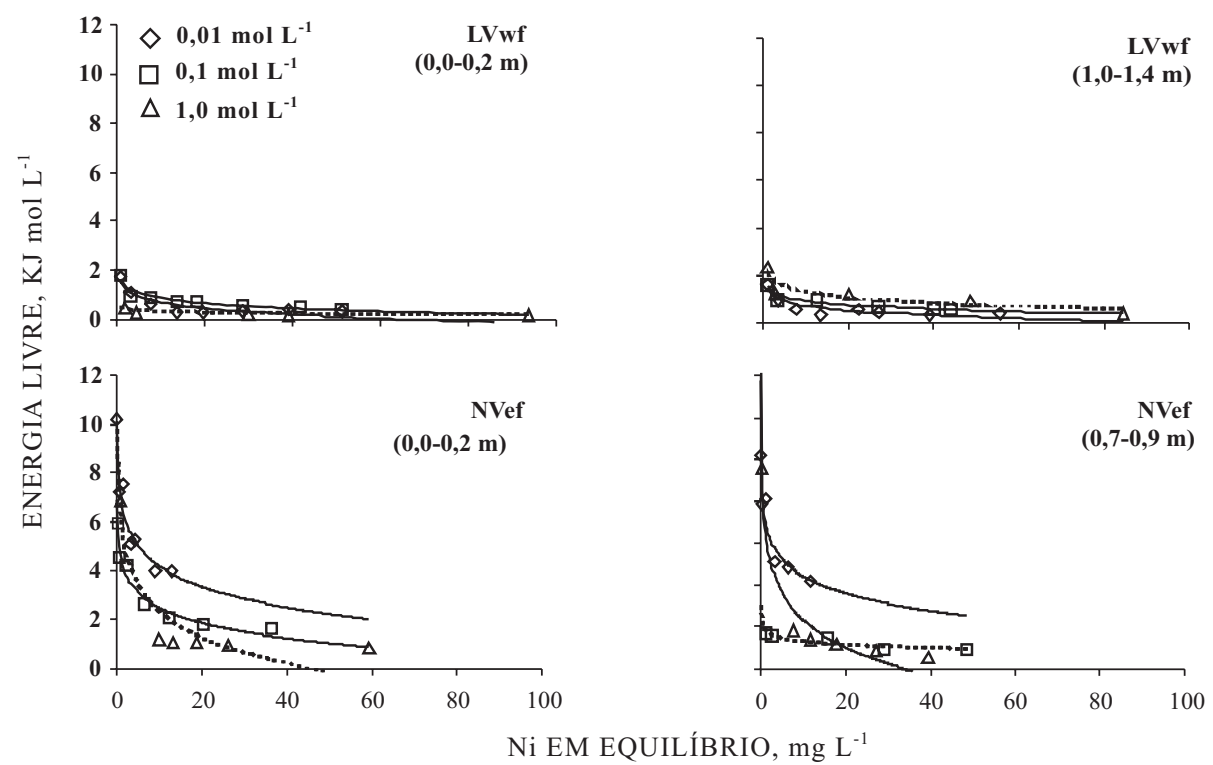

Figura 2. Energia livre da reação de adsorção de Ni para Latossolo Vermelho acriférrico (LVwf) e Nitossolo Vermelho eutroférrico (NVef), coletados em superfície e profundidade, utilizando $\mathrm{CaCl}_{2}$ nas forças iônicas 0,$01 ; 0,1$; e $1,0 \mathrm{~mol} \mathrm{~L}^{-1}$.

A energia livre da reação de adsorção de Ni foi maior quando se utilizou $\mathrm{NaCl}$ (Figura 1) como eletrólitosuporte do que $\mathrm{CaCl}_{2}$ (Figura 2). Esse efeito pode ser atribuído à maior competição entre $\mathrm{Ni}^{2+} \mathrm{e} \mathrm{Ca}^{2+}$ por sítios de adsorção e à elevada especificidade do íon $\mathrm{Ca}^{2+}$ e seu efeito na espessura da dupla camada difusa, já que o potencial no plano de adsorção do colóide está relacionado com a valência do íon, o que afeta a densidade de carga superficial. Assim, aumentandose a valência do cátion, o potencial da superfície adsorvente torna-se menos negativo em valores de $\mathrm{pH}$ maiores que o ponto de carga zero do solo, o que reduz a adsorção de cátions (Naidu et al., 1994).

Quando se utilizou o $\mathrm{NaCl}$, os valores de energia livre no LVwf foram maiores em todas as forças iônicas desse eletrólito, demonstrando que nesse solo a contribuição das cargas elétricas no fenômeno adsortivo é maior. No NVef esse comportamento também foi observado, porém os valores de $\Delta \mathrm{G}_{0}$ obtidos com os dois eletrólitos foram semelhantes (Figuras 1 e 2). Destaca-se, assim, a relevância de se considerar a força iônica do meio como forma de regular a disponibilidade do elemento. A adsorção de metais depende conjuntamente da força iônica, da composição do eletrólito-suporte e da natureza das reações envolvidas (Harter \& Naidu, 2001). Tendo em vista que o $\mathrm{Ca}^{2+}$ pode competir mais fortemente que o $\mathrm{Na}^{+}$ por sítios de ligação, a extensão na qual a força iônica irá alterar a adsorção de metais por solos oxídicos vai depender do íon dominante na solução do solo (Ca ou $\mathrm{Na}$.

Observou-se maior energia livre da reação de adsorção de Ni no NVef do que no LVwf, o que está relacionado com a sua textura mais argilosa, maior
SE e balanço de cargas negativo em todo o seu perfil (Silveira et al., 1999; Mellis et al., 2004), além dos maiores valores de cargas negativas variáveis $\left(\sigma_{\mathrm{H}}\right)$ e permanentes $\left(\sigma_{0}\right)$ do NVef. Nesses solos, $\sigma_{\mathrm{H}}$ foi igual a 63,1 e $\sigma_{0}$ foi igual a 30,2 mmol $_{\mathrm{c}} \mathrm{kg}^{-1}$ na camada de $0-0,2 \mathrm{~m}$, contra $\sigma_{\mathrm{H}}=39,9$ e $\sigma_{0}=8,2$ para o LVwf, na mesma camada (Silveira et al., 2002). Em solos como o NVef, os principais minerais responsáveis pela geração de cargas são a MO, a caulinita e os óxidos de $\mathrm{Fe}$ e Al, cujas cargas são variáveis com o pH e com a força iônica do meio.

A energia livre foi maior nas camadas superficiais do que nas subsuperficiais (Figuras 1 e 2). A MO deve ter sido o principal fator responsável por esse comportamento, concordando com os resultados obtidos por Silveira et al. (1999) e Dias et al. (2003). Apesar de ocorrer em teores bem mais baixos que o de argilominerais, a MO é o fator ponderável na CTC da camada superficial de solos paulistas, representando, em média, 70 \% do total (Raij, 1969). A importância da MO na disponibilidade de metais pesados tem sido enfatizada por muitos autores (Alleoni, 2000; Yin et al., 2002), mas existem aqueles que acreditam que tal importância venha sendo superestimada, visto que o conteúdo de MO total do solo nem sempre está relacionado à sua porção reativa que irá efetivamente interagir com o metal (Harter, 1979, 1983; Bloomfield, 1981).

Mellis et al. (2004), estudando o efeito da MO e outros atributos na adsorção de Ni por três solos, entre eles o LVwf e o NVef, notaram que o efeito da presença da MO alterou de forma evidente a adsorção do metal. Para as amostras de LVwf e NVef sem MO, a adsorção média de Ni foi, respectivamente, 11 e $28 \%$ menor do 
que aquela obtida para as amostras originais dos mesmos solos. Além desse atributo, o $\Delta \mathrm{pH}$ mais negativo e o PESN menor em superfície, em conseqüência do conteúdo elevado de MO, também contribuíram para maior adsorção de Ni nas camadas superficiais das amostras.

\section{CONCLUSÕES}

1. A energia livre de adsorção de Ni diminuiu com o aumento da concentração de Ni adicionada.

2. O Nitossolo Vermelho eutroférrico apresentou maior adsorção de $\mathrm{Ni}$ e, portanto, maior energia livre da reação que o Latossolo Vermelho acriférrico.

3. A energia livre da reação foi maior nos horizontes superficiais devido aos maiores teores de matéria orgânica.

4. A energia livre foi maior para as menores forças iônicas do meio, tanto para $\mathrm{CaCl}_{2}$ quanto para $\mathrm{NaCl}$, com destaque para o último.

5. As reações de adsorção de Ni são espontâneas e exotérmicas, mesmo no Latossolo ácrico, com balanço positivo de cargas.

\section{AGRADECIMENTO}

À Fundação de Apoio à Pesquisa do Estado de São Paulo (FAPESP), pela concessão da bolsa de Iniciação Científica à primeira autora.

\section{LITERATURA CITADA}

ALLEONI, L.R.F. \& CAMARGO, O.A. Energia livre das reações de adsorção de boro em solos. R. Agric., 73:319-330, 1998.

ALLEONI, L.R.F. Química de Latossolos ácricos. Piracicaba, Escola Superior de Agricultura "Luiz de Queiroz", 2000. 121p. (Tese de Livre-Docência)

ALLEONI, L.R.F. \& CAMARGO, O.A. Atributos físicos de Latossolos ácricos do norte paulista. Sci. Agric., 51:321326, 1994.

ALLEONI, L.R.F. \& CAMARGO, O.A. Óxidos de ferro e de alumínio, e mineralogia da fração argila deferrificada de latossolos ácricos. Sci. Agric., 53:416-421, 1995.

BLOOMFIELD, C. The translocation of metal in soil. In: GREENLAND, D.J. \& HAYES, M.H., eds. The chemistry of soil processes. New York, John Wiley \& Sons, 1981. $714 \mathrm{p}$.

CAMARGO, O.A.; MONIZ, A.C.; JORGE, J.A. \& VALADARES, J.M.A. Métodos de análise química, mineralógica e física de solos do IAC. Campinas, Instituto Agronômico, 1986. 94p. (Boletim Técnico, 106)
CAMARGO, O.A.; ROVERS, H. \& VALADARES, J.M.A.S. Adsorção de níquel em latossolos paulistas. Bras. Ci. Solo, 13:125-129, 1989

CASAGRANDE, J.C.; ALLEONI, L.R.F.; CAMARGO, O.A. \& ARNONE, A.D.M. Ionic strength, $\mathrm{pH}$ and zinc adsorption in a variable charge soil. Comm. Soil Sci. Plant Anal., 33:28-35, 2002.

CASAGRANDE, J.C.; JORDÃO, C.B.; ALLEONI, L.R.F. \& CAMARGO, O.A. Copper desorption in a soil with variable charge. Sci. Agric., 61:196-202, 2004.

CIHACEK, J.L. \& BREMNER, J.M. A simplified ethylene glycol monoethyl procedure for assessment of soil surface area. Soil Sci. Soc. Am. J., 43:821-822, 1979

DIAS, N.M.P.; ALLEONI, L.R.F.; CASAGRANDE, J.C. \& CAMARGO, O.A. Energia livre da reação de adsorção de cádmio em Latossolo ácricos. Ci. Rural, 33:829-834, 2003.

EMPRESA BRASILEIRA DE PESQUISA AGROPECUÁRIA EMBRAPA. Sistema brasileiro de classificação de solos. 2.ed. Rio de Janeiro, 2000. 412p.

HARTER, R.D. Adsorption of copper and lead by Apand B2 horizons of several Northeastern United States soils. Soil Sci. Soc. Am. J., 43:679-683, 1979.

HARTER, R.D. Effect of soil $\mathrm{pH}$ on adsorption of lead, copper, zinc and nickel. Soil Sci. Soc. Am. J., 47:47-51, 1983.

HARTER. R.D. \& NAIDU, R. An assessment of environmental and solution parameter impact on trace-metal sorption by soils. Soil Sci. Soc. Am. J., 65:597-612, 2001.

HEILMAN, M.D.; CARTER, D.L. \& GONZALEZ, C.L. The ethylene glycol monoethyl (EGME) technique for determining soil-surface area. Soil Sci., 100:409-413, 1965.

MELLIS, E.V.; CRUZ, M.C.P. \& CASAGRANDE, J.C. Nickel adsorption by soils in relation to $\mathrm{pH}$, organic matter, and iron oxides. Sci. Agric., 61:190-195, 2004.

MIRANDA-TREVINO, J.C. \& COLES, C.A. Kaolinite properties, structure and influence of metal retention on pH. Appl. Clay Sci., 23:133-139, 2003.

NAIDU, R.; BOLAN, N.S.; KOOKANA, R.S. \& TILLER, K.G. Ionic-strength and $\mathrm{pH}$ effects on the sorption of cadmium and the surface charge of soils. Eur. J. Soil Sci., 45:419429, 1994.

OLIVEIRA, K.W.; MELO, W.J.; PEREIRA, G.T.; MELO, V.P. \& MELO, G.M.P. Heavy metals in Oxisols amended with biosolids and cropped with maize in a long-term experiment. Sci. Agric., 62:381-388, 2005.

PERCIVAL, H.J.; SPEIR, T.W. \& PARSHOTAM, A. Soil solution chemistry of contrasting soils amended with heavy metals. Aus. J. Soil Res., 37:993-1004, 1999.

POMBO, L.; SALGADO, V.; VOLKWEISS, S. \& KLAMT, E. Adsorção de níquel por dois solos: Terra bruna estruturada similar e Podzólico Vermelho-Amarelo. Pesq. Agropec. Bras., 24:593-598, 1989.

PROCTOR, J. \& BAKER, A.J.M. The importance of nickel for plant growth in ultramafic (serpentine) soils. In: ROSS, S., ed. Toxic metals in soil-plant systems. New York, Jonh Wiley, 1994. p.417-432. 
RAIJ, B. van \& PEECH, M. Electrochemical properties of some Oxisols and Alfisols of the tropics. Soil Sci. Soc. Am. Proc., 36:587-593, 1972.

RAIJ, B. van. A capacidade de troca de cátions das frações orgânicas e mineral do solo. Bragantia, 28:85-112, 1969.

RESENDE, M. \& SANTANA, D.P. Uso das relações Ki e Kr na estimativa da mineralogia para classificação dos Latossolos. In: REUNIÃO DE CLASSIFICAÇÃO, CORRELAÇÃO DE SOLOS E INTERPRETAÇÃO DE APTIDÃO AGRÍCOLA, 3., Rio de Janeiro, 1988. Anais. Rio de Janeiro, Embrapal SNLCS, 1988. p.225-232.

ROVERS, H.; CAMARGO, O.A. \& VALADARE, J.M.A.S. Níquel total e solúvel em DTPA em solos do estado de São Paulo. R. Bras. Ci. Solo, 7:217-220, 1983.

SCHEIDEGGER, A.M.; FENDORF, M. \& SPARKS, D.L. Mechanisms of nickel sorption on pyrophyllite: Macroscopic and microscopic approaches. Soil Sci. Soc. Am. J., 60:1763-1772, 1996.
SILVEIRA, M.L.A.; ALLEONI, L.R.F.; CASAGRANDE, J.C. \& CAMARGO, O.A. Energia livre da reação de adsorção de cobre em Latossolo ácricos. Sci. Agric., 56:1117-1122, 1999.

SILVEIRA, M.L.A.; ALLEONI, L.R.F.; CASAGRANDE, J.C. \& CAMARGO, O.A. Copper adsorption in oxidic soils after removal of organic matter and iron oxides. Comm. Soil Sci. Plant Anal., 33:3581-3592, 2002.

SINGH, M. Retention and added cooper by two soils as affected by organic matter, $\mathrm{CaCO}_{3}$ and exchangeable ions. Equilibrium adsorption of boron in soils and clays. Geoderma, 5:2019-2217, 1971.

TEIXEIRA, S.T.; MELO, W.J. \& SILVA, E.T. Heavy metals in a degraded soil treated with sludge from water treatment plant. Sci. Agric., 62:498-501, 2005.

UREN, N.C. Forms, reactions, and availability of nickel in soils. Adv. Agron., 48:141-203, 1992. 